\title{
Metabolic recovery of Arabidopsis thaliana roots following cessation of oxidative stress
}

\author{
Martin Lehmann • Miriam Laxa • \\ Lee J. Sweetlove · Alisdair R. Fernie • \\ Toshihiro Obata
}

Received: 16 December 2010/ Accepted: 28 February 2011/Published online: 12 March 2011

(C) The Author(s) 2011. This article is published with open access at Springerlink.com

\begin{abstract}
To cope with the various environmental stresses resulting in reactive oxygen species (ROS) production plant metabolism is known to be altered specifically under different stresses. After overcoming the stress the metabolism should be reconfigured to recover basal operation however knowledge concerning how this is achieved is cursory. To investigate the metabolic recovery of roots following oxidative stress, changes in metabolite abundance and carbon flow were analysed. Arabidopsis roots were treated by menadione to elicit oxidative stress. Roots were fed with ${ }^{13} \mathrm{C}$ labelled glucose and the redistribution of isotope was determined in order to study carbon flow. The label redistribution through many pathways such as glycolysis, the tricarboxylic acid (TCA) cycle and amino acid metabolism were reduced under oxidative stress. After menadione removal many of the stress-related changes reverted back to basal levels. Decreases in amounts of hexose phosphates, malate, 2-oxoglutarate, glutamate and aspartate were fully recovered or even increased to above the control level. However, some metabolites such as pentose phosphates and citrate did not recover but maintained their levels or even increased further. The alteration in label redistribution largely correlated with that in metabolite abundance. Glycolytic carbon flow reverted to
\end{abstract}

Electronic supplementary material The online version of this article (doi:10.1007/s11306-011-0296-1) contains supplementary material, which is available to authorized users.

M. Lehmann · A. R. Fernie $(\bowtie) \cdot$ T. Obata

Max-Planck-Institute of Molecular Plant Physiology,

Am Mühlenberg 1, 14476 Potsdam-Golm, Germany

e-mail: Fernie@mpimp-golm.mpg.de

M. Laxa · L. J. Sweetlove

Department of Plant Sciences, University of Oxford,

South Parks Road, Oxford OX1 3RB, UK the control level only $18 \mathrm{~h}$ after menadione removal although the TCA cycle and some amino acids such as aspartate and glutamate took longer to recover. Taken together, plant root metabolism was demonstrated to be able to overcome menadione-induced oxidative stress with the differential time period required by independent pathways suggestive of the involvement of pathway specific regulatory processes.

Keywords Oxidative stress - Menadione - Recovery · Metabolic flux $\cdot$ Arabidopsis thaliana $\cdot$ Metabolite profiling

\section{Introduction}

Plants are sessile organisms and as such have limited possibilities to avoid non-optimal environmental conditions. When they are exposed to various stressful factors such as heat, frost, drought, flooding, high irradiation, shading, osmotic disorder or herbivorous attack, such stresses affect plant metabolism. It is necessary for plants to adapt to the new situation via so-called "stress responses". Independently from the nature of stress, a common consequence is an imbalance of the production and the scavenging of reactive oxygen species (ROS) resulting in oxidative stress (Schutzendubel and Polle 2002). An uncontrolled increase of ROS is fatal for the plant since they can disrupt the sensitive redox system, damage proteins, lipids and DNA and lead to a cellular dysfunction and cell death (Møller et al. 2007). To cope with oxidative stress plants have developed highly effective metabolic mechanisms for ROS detoxification. One is the presence of multiple redox buffer systems. Plants employ ascorbate and glutathione as major cellular redox buffers as well as tocopherols, flavonoids, alkaloids and carotenoids. Plants also 
involve a set of scavenging enzymes such as superoxide dismutase, ascorbate peroxidase, glutathione peroxidase and catalase (Apel and Hirt 2004). Furthermore, it has been shown that the plant metabolism is reorganised under different stress conditions, e.g. salt, cold and oxidative stress (Gong et al. 2005; Kaplan et al. 2007; Lehmann et al. 2009). This allows the plants to prevent the accumulation of ROS and to produce indispensable metabolites. The metabolomic approach has been proven as a powerful tool to gain an overview of such metabolic reorganisation in response to stressful conditions (reviewed in Shulaev et al. 2008).

In a previous study, metabolic and transcriptomic responses were analysed in menadione treated Arabidopsis roots (Lehmann et al. 2009). Menadione is a quinone which causes ROS generation from the mitochondrial and plastidal electron transport chains. Given its hydrophobic properties it can pass biological membranes easily to reach these organelles and releases ROS such as superoxide, hydrogen peroxide and hydroxyl radicals (Castro et al. 2007; Chung et al. 1999; Mauzeroll et al. 2004). Detailed metabolic studies revealed a down-regulation of glycolysis and tricarboxylic acid (TCA) cycle and the redirection of carbon metabolism from glycolysis to oxidative pentose phosphate pathway (OPPP). Furthermore, an increase of $O$-acetyl serine (OAS), $\gamma$-aminobutyric acid (GABA) and many proteogenic amino acids was observed. At the transcriptional level a broad down-regulation of the genes related to central carbon metabolism were detected after $2 \mathrm{~h}$ of stress and it levelled off after $6 \mathrm{~h}$. These results thus suggest that plant root metabolism had adapted to menadione-induced oxidative stress and had already carried out a "recovery response" by this time point.

Given the fluctuation of the natural environment most of stresses are not persistent and the plant thus needs to act flexibly. Alongside being able to provide a rapid and effective response to various stresses the plant equally needs to rapidly reconstruct its basal physiology on cessation of the stressful situation. This recovery response is necessary to allow the plant to be able to cope with future stresses in an appropriate manner and is important to circumvent the energy costs required to maintain the operation of metabolism unfavourable under normal growth condition. Recovery responses after various stresses have been observed and analysed in several previous studies. For example a decrease in catalase activity in the desert plant, Reaumuria soongorica, as a result of ongoing drought and oxidative stress was able to be recovered after the stress treatment was ceased. On the other hand, guaiacol peroxidase, showing a similar stress response, could not recover from its inhibition (Bai et al. 2009). In Arabidopsis plants the cellular redox state is affected by externally supplied $\mathrm{H}_{2} \mathrm{O}_{2}$ but recovered quickly after the stress removal
(Schwarzlander et al. 2009). Although all studies provide details as to how plant metabolism can respond following a stress event, comprehensive metabolite profiling and flux studies on stress and stress recovery responses are still missing.

In this study we investigated changes in both metabolite abundance and isotope redistribution during and following menadione treatment in the root system of Arabidopsis thaliana. To examine the stress responses the plants received a short term oxidative stress of $6 \mathrm{~h}$ and then the stress was removed and the recovery response was analysed over the following $24 \mathrm{~h}$. In addition to metabolite profiling, an isotope redistribution analysis was conducted in order to complete our overview of the metabolic responses since metabolite abundances alone do not always reflect changes in flux through pathways. Actually in Arabidopsis cultured cells the down-regulation of the glycolysis and TCA cycle under oxidative stress were detectable only by ${ }^{13} \mathrm{C}$-carbon isotope redistribution analysis (Baxter et al. 2007a). A flux analysis at the metabolic fraction level (e.g. total amino acids and total organic acids) performed in the previous study also did not fit to uncover the metabolic response. This is likely because a change of metabolic flux on a metabolite can be counteracted by that of other metabolites in the same fraction. In the present study, we performed a isotope redistribution analysis on the single metabolite level for both stress and recovery phases. Furthermore, we investigated the activity of several key enzymes of the glycolysis and the TCA cycle. By employing these analytical methods we tried to provide an integrated and comparative view of the stress and stress recovery responses of Arabidopsis root metabolism. Many metabolic "recovery" responses were observed in practice after the secession of oxidative stress and the various time needed for the recovery of each pathway suggested the involvement of specific regulatory mechanism for individual pathways. The fast reactivation of a pathway such as glycolysis by a particular mechanism uncoupled from the enzymatic activity might contribute to the plant survival by assuring production of energy and metabolites when other pathway is inhibited severely.

\section{Materials and methods}

\subsection{Plant cultivation and oxidative stress treatment}

Arabidopsis thaliana Col-0 plants were germinated on agar and grown hydroponically as described in Lehmann et al. (2009). Plants were placed on the cover of black plastic containers filled with 101 of nutrient solution (Gibeaut et al. 1997) which was exchanged twice a week. The photoperiod was $12 \mathrm{~h}$ for both day and night with 
$100 \mu \mathrm{mol} \mathrm{m}{ }^{-2} \mathrm{~s}^{-1}$ light intensity. The growth temperature was 20 and $18{ }^{\circ} \mathrm{C}$ and the relative humidity was 75 and $85 \%$ at the day and the night, respectively. Oxidative stress was induced in the 4.5 week old plants by adding six millilitre of $100 \mathrm{mM}$ menadione dissolved in ethanol into the nutrient solution. To perform the recovery experiment the menadione was removed after $6 \mathrm{~h}$ incubation time by exchanging the nutrient solution. The roots were briefly washed by dipping repeatedly in a separate tank containing fresh nutrient solution and then placed into fresh tanks. Control plants were treated in the same way with the equivalent concentration of ethanol. Root tissue was harvested at $0,0.5,2$ and $6 \mathrm{~h}$ after the stress induction and at two additional time points, 24 and $30 \mathrm{~h}$, for the recovery analyses. The scheme of experimental design is shown in Suppl. Fig. S1A. Harvested roots were frozen immediately in liquid nitrogen and stored at $-80^{\circ} \mathrm{C}$ for metabolite profiling and enzyme assays.

\section{$2.2{ }^{13} \mathrm{C}$ labelling}

To estimate the carbon flow through the central metabolism we performed a ${ }^{13} \mathrm{C}$-redistribution analysis by feeding $50 \mathrm{mM}$ of glucose labelled with the ${ }^{13} \mathrm{C}$ stable isotope in all carbon atoms. The plants were grown as described above and were transferred 1 day before the experiment to the mini hydroponic system using $4 \mathrm{ml}$ plastic tubes covered with aluminium foil keeping the roots in the dark. The ${ }^{13} \mathrm{C}$-glucose was added to the hydroponic solution $3 \mathrm{~h}$ prior to the harvest at each time point to keep a constant labelling period ensuring logarithmic increase of label in each metabolite (Suppl. Fig. S1B). Roots were harvested at 0, $0.5,2,6 \mathrm{~h}$ after the addition of menadione. For the recovery experiment, the nutrient solution was exchanged after $6 \mathrm{~h}$ of menadione treatment against fresh one. The ${ }^{13} \mathrm{C}$-redistribution on each metabolite was evaluated by using a gas chromatography-time of flight-mass spectrometry (GC-TOF-MS) as follows.

\subsection{Mass spectrometry based metabolite analysis}

The metabolites were analysed by a GC-TOF-MS (Pegasus III, Leco, St. Joseph, USA). The material was prepared from five replicates of samples and analysed as described in Roessner et al. (2001) and Lisec et al. (2006) with slight modifications. The exact procedure is given in Lehmann et al. (2009). As an internal standard $3.2 \mu \mathrm{g} / \mathrm{ml}$ of ribitol was added to each sample prior to the extraction.

\subsection{Calculation of ${ }^{13} \mathrm{C}$ label redistribution}

For the calculation of the label redistribution, the proportion of the ${ }^{13} \mathrm{C}$ in specific mass fragments of the metabolites was determined. When ${ }^{13} \mathrm{C}$ accumulated in a metabolite pool, mass fragments containing ${ }^{13} \mathrm{C}$ have a mass shift with $+1 \mathrm{~m} / \mathrm{z}$ for each ${ }^{13} \mathrm{C}$. The molar percentage enrichments were evaluated by determination of the intensities of the ${ }^{12} \mathrm{C}$ spectral fragments, and the isotopic spectral fragments of nonlabeled standards were compared with the fragmentation patterns of metabolites detected in the chromatograms of the ${ }^{13} \mathrm{C}$-fed samples as described in Giegé et al. (2003). The ${ }^{13} \mathrm{C}$ label redistribution was calculated according to RoessnerTunali et al. (2004). To calculate the redistribution of the label the ${ }^{13} \mathrm{C}$ label enrichment was multiplied by the total amount of the metabolite calculated from a calibration curve for each compound and divided by the time of labelling $(3 \mathrm{~h})$ and the molarity of ${ }^{13} \mathrm{C}$ label supplied $(50 \mathrm{mM})$.

\subsection{Enzyme activity assays}

For the determination of total cellular activities of the enzymes, namely citrate synthase (CSY), $\mathrm{NAD}^{+}$dependent isocitrate dehydrogenase (IDH), aconitase (ACO), malate dehydrogenase (MDH), glucoso-6-phosphate isomerase (PGI), phosphofructo kinase (PFK), triosophosphate isomerase (TPI), glyceraldehyde-3-phosphate dehydrogenase (GAPDH), 3-phospho-glycerate kinase (PGK), enolase (ENO), and pyruvate kinase (PK), $100 \mathrm{mg}$ of homogenised frozen root material was used. The enzymes were extracted in $500 \mu \mathrm{l}$ of extraction buffer (50 mM 4-(2-2-hydroxyethyl)1-piperazineethanesulfonic acid- $\mathrm{KOH}, \mathrm{pH}$ 7.4, $5 \mathrm{mM}$ $\mathrm{MgCl}_{2}, 1 \mathrm{mM}$ ethylenediaminetetraacetic acid, $1 \mathrm{mM}$ ethyleneglycoltetraacetic acid, $0.25 \mathrm{mM}$ dithiothreitol, $0.1 \%$ (v/v) Triton X-100, 0.1\% BSA, $0.5 \mathrm{mM}$ phenylmethylsulfonyl fluoride, $2 \mathrm{mM}$ benzamidine and $2 \mathrm{mM}$-aminocaproic acid) and centrifuged at $20,000 \times g$ at $4^{\circ} \mathrm{C}$ for $5 \mathrm{~min}$. The supernatant was desalted by using an Illustra NAP-5 column (GE Healthcare, Buckinghamshire, UK) equilibrated with extraction buffer without protease inhibitors. The activities of CSY, IDH, ACO and MDH were measured as described in Jenner et al. (2001). The activities of PGI, PFK, TPI, GAPDH, PGK, ENO, and PK were measured according to Brandina et al. (2006). Enzyme extraction and activity assay of fumarase (FUM) were performed as described in Gibon et al. (2004).

\subsection{Chemicals}

All chemicals were purchased from Sigma-Aldrich (St. Louis, MO), Merck (Darmstadt, Germany) or Duchefa (Haarlem, Netherlands). The ${ }^{13} \mathrm{C}$-glucose was provided by Cambridge Isotope Laboratories (Andover, USA). Enzymes used for enzyme activity assays were purchased from Roche (Basel, Switzerland). 


\subsection{Data analysis}

All values obtained by GC-MS technique were normalised by fresh weight and the intensity of internal standard for relative quantification. Data were analysed by the $t$-test function in Excel (Excel 2003, Microsoft, Redmond, USA). The MapMan software was used to create the heatmap (Usadel et al. 2005). The data were centred by the average of the controls to create heatmap.

\section{Results}

\subsection{The metabolic carbon flow under oxidative stress}

The metabolite abundance indicates the changes in metabolic activity of a pathway. Nevertheless, the carbon flow can be affected without any apparent changes in the metabolite pool sizes (Baxter et al. 2007a; Fell 2005; Fernie et al. 2005). Taken this into account, flux analysis is necessary to get a comprehensive overview of the metabolic response. We conducted ${ }^{13} \mathrm{C}$-redistribution analysis under menadioneinduced oxidative stress to complete the observations provided by our previous study (Lehmann et al. 2009). In total 48 polar metabolites such as intermediates of the glycolysis, the TCA cycle and many amino acids could be annotated and analysed although those of the OPPP were not detected (Fig. 1, Suppl. Figs. S2-S4). Menadione treatment caused changes in the carbon redistribution of the glycolytic intermediates namely glucose-6-phosphate (G6P) and fructose-6phosphate (F6P). Both were temporary reduced after $2 \mathrm{~h}$ of oxidative stress although the change in F6P was not statistically significant (Fig. 1, Suppl. Fig. S2). On the other hand, the ${ }^{13} \mathrm{C}$-redistribution of pyruvate increased strongly after the same stress period of $2 \mathrm{~h}$. Interestingly, these effects were not obvious anymore following $6 \mathrm{~h}$ of oxidative stress. Almost all intermediates of the TCA cycle, except succinate, displayed a reduction of the label redistribution after 2 and $6 \mathrm{~h}$ of oxidative stress (Fig. 1, Suppl. Fig. S3). The ${ }^{13} \mathrm{C}$ redistribution in aspartate and glutamate, derived from TCA cycle intermediates, also decreased dramatically over the whole stress period. The carbon flow in other amino acids such as ornithine and proline increased at least temporarily although those of serine and tyrosine were decreased. However, many amino acids such as alanine, asparagine, glutamine, methionine and lysine did not show any significant response in ${ }^{13} \mathrm{C}$-redistribution. The label redistribution to OAS, a precursor of the sulphur containing amino acids, increased significantly after $2 \mathrm{~h}$ of menadione treatment although after $6 \mathrm{~h}$ the effect was strongly reduced (Fig. 1, Suppl. Fig. S4).
3.2 Changes in metabolite abundance after the removal of oxidative stress

To assess how plant root metabolism recovers following the cessation of the oxidative stress we performed a comparative metabolic analysis. After $6 \mathrm{~h}$ of stress application, menadione was removed and the plants were grown, free from stressor, for the following $24 \mathrm{~h}$. The plants were harvested at $0,6,24$ and $30 \mathrm{~h}$ after the addition of menadione. Both time points of 24 and $30 \mathrm{~h}$ represent the recovery phase (Suppl. Fig. S1). In total 66 polar metabolites were detected and the menadione induced changes in metabolite levels after $6 \mathrm{~h}$ were comparable with those shown in our previous study (Lehmann et al. 2009). The response of decreasing levels of hexose phosphates, succinate, fumarate, malate, glutamate and aspartate were observed in both experiments as were the increases in the levels of pyruvate, citrate, 2-oxo-glutarate (2OG), OAS and many amino acids. During the recovery phase the levels of these metabolites returned back to control levels (Fig. 1, Suppl. Figs. S5-S7). The most drastic recovery responses were observed for the hexose phosphates. Following the removal of menadione they increased strongly above the control level, suggesting a reactivation of the glycolytic pathway. Almost all detected sugars also displayed a recovery response and increased after stress removal. Glucose and fructose increased only after the stress was removed (Fig. 1, Suppl. Fig. S5). A very similar behaviour was found in raffinose (Fig. 1, Suppl. Fig. S5), known as stress marker metabolite (Van den Ende and Valluru 2009). We could not quantify the amount of sucrose accurately in the menadione treated samples because this peak was overloaded at all time points, suggesting an enormous increase under oxidative stress. The pentose phosphates, ribulose- and ribose-5-phosphate, showed no recovery response. Ribulose-5-phosphate increases further after menadione was removed and ribose-5-phosphate decreased slightly during the recovery phase (Fig. 1, Suppl. Fig. S5). Pyruvate, a metabolite linking glycolysis and the TCA cycle, accumulated enormously during the stress period but reduced its level markedly in the recovery phase. The pyruvate level was still significantly higher than the control plants but showed a clear recovery effect (Fig. 1, Suppl. Fig. S6).

In addition stress responses of many TCA cycle intermediates were observed (Fig. 1, Suppl. Fig. S6). Citrate, one of the intermediates of the decarboxylating part of the TCA cycle, increased very strongly and rapidly after the stress removal. 2OG increased at $6 \mathrm{~h}$ of the stress treatment and decreased after the stress removal. The pool size was reduced to the half within $18 \mathrm{~h}$ after the menadione was removed although it is still twice as much as that in the 


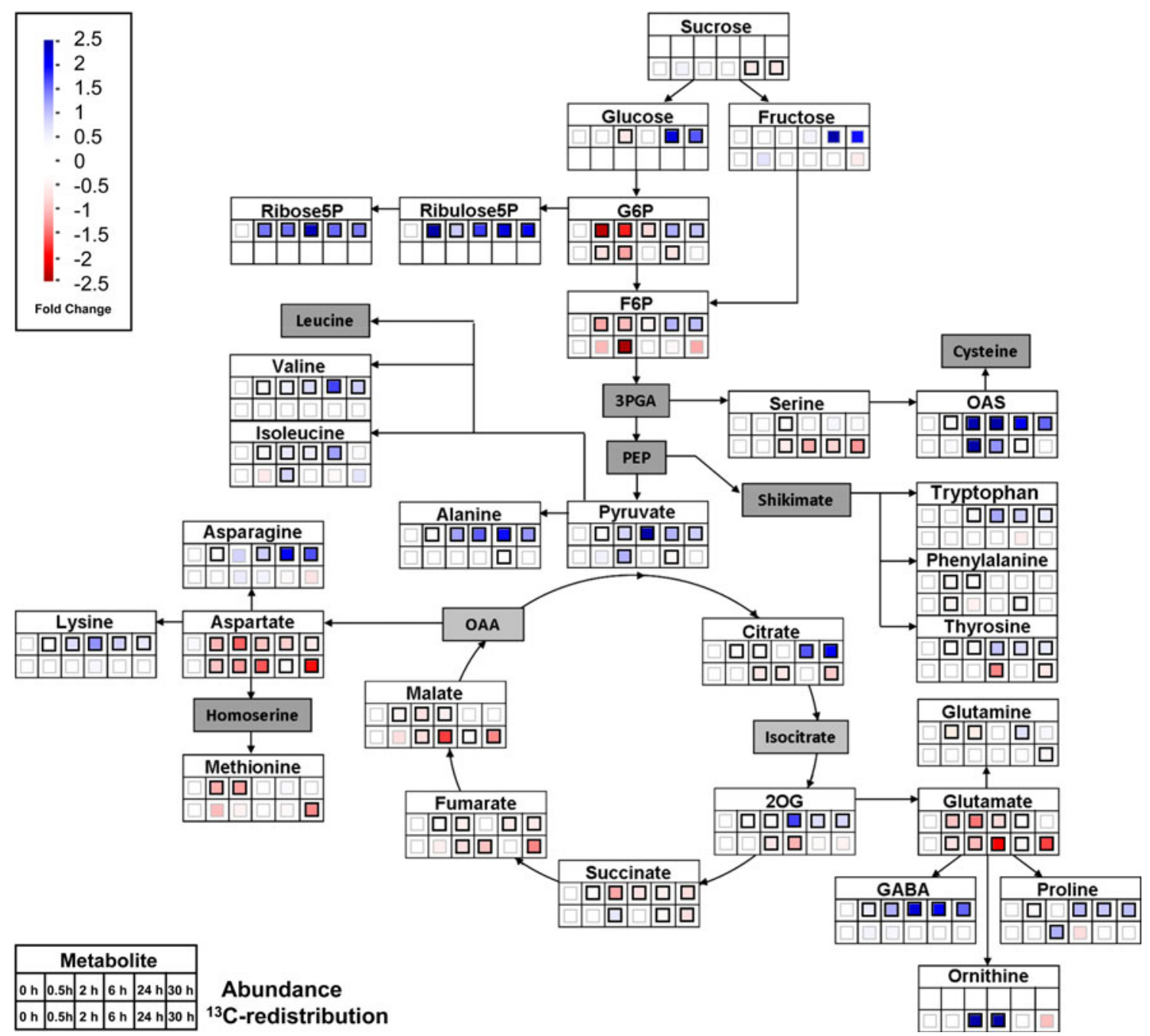

Fig. 1 Scheme of metabolite abundance and carbon flow changes during menadione induced oxidative stress and recovery. Arabidopsis roots were treated with menadione for $6 \mathrm{~h}$ and then menadione was removed from growth media. The first four time points $(0,0.5,2$ and $6 \mathrm{~h}$ ) represent the stress period and the last time points $(24$ and $30 \mathrm{~h})$ represent the recovery phase. The relative metabolite abundance and ${ }^{13} \mathrm{C}$-redistribution in menadione treated roots against control roots are shown as a heatmap. The metabolite abundance changes are presented in the first row and the metabolic carbon flow changes are presented

control plants. Succinate was significantly decreased under oxidative stress and the level was kept low in the recovery phase with a clear trend of increase. Fumarate started to decrease within the $6 \mathrm{~h}$ of stress period and decreased continuously until the $24 \mathrm{~h}$ harvesting time point and then kept the same level until $30 \mathrm{~h}$. In contrast to succinate and fumarate, which responded more moderately, malate demonstrated a very clear and distinct stress recovery response. After the stress application the malate decreased significantly and recovered quickly onto control plant level in the recovery phase (Fig. 1, Suppl. Fig. S6). Taken together, metabolite abundance in the decarboxylating part in the second row. Red and blue colours characterise decreasing and increasing values in menadione treated plants, respectively. The metabolites which revealed significant changes in $t$-test $(P<0.05)$ were shown in bold box. Change of each metabolite is shown in Suppl. Figs. S2-S7. The metabolites at the empty positions were not determined. Metabolites highlighted in grey were not annotated in the experiments. The data of the metabolite abundances for the 0.5 and $2 \mathrm{~h}$ time points are taken from the previous study (Lehmann et al. 2009), performed under equal conditions (Color figure online)

of the TCA cycle increased and the non-decarboxylating part decreased under oxidative stress and $2 \mathrm{OG}$ and malate showed clear and distinct recovery within $24 \mathrm{~h}$ from stress removal in contrast to other intermediates such as succinate and fumarate those showed trend of recovery but probably needed more time to reach to the control level.

The GABA shunt is considered to be important in plants to support the metabolic flow under stress conditions by bypassing the decarboxylating steps of TCA cycle from $2 \mathrm{OG}$ to succinate (Fait et al. 2008). As already mentioned above the 2OG showed an explicit stress response and normalised quickly afterwards. Glutamate, the first 
intermediate in GABA shunt, exposed a behaviour negatively correlated to $2 \mathrm{OG}$ and decreased to one-third of the unstressed level. At the $24 \mathrm{~h}$ time points the difference between the treated and control plants were already much smaller than the end of stress phase and at $30 \mathrm{~h}$ of recovery there was no significant difference anymore. GABA was increased strongly under stress and showed a slow but clear tendency for a subsequent reduction (Fig. 1, Suppl. Fig. S7). Succinate responded as stated above.

Furthermore, we detected clear recovery in many amino acids (Fig. 1, Suppl. Fig. S7). Aspartic acid, synthesised by using oxaloacetate as carbon skeleton, was decreased under oxidative stress and behaved similarly to glutamate. Both metabolites are related to the first steps of nitrogen fixation, which has been known since the 1960s (Virtanen 1961). Other amino acids primarily derived from glutamate like proline, as well as those derived from aspartic acid such as asparagine, lysine and threonine showed the opposite behaviour. The levels of these amino acids increased under stress and showed a clear trend to decrease after the stress was removed. Some other amino acids such as the aromatic amino acids especially tryptophan and tyrosine and amino acids basically synthesised from pyruvate namely alanine, valine and isoleucine also responded in the same way (Fig. 1, Suppl. Fig. S7). Additionally OAS increased dramatically in all treated samples under menadione treatment (although the level was almost as low as the detection limit in the control samples). The OAS content showed a peak at the $6 \mathrm{~h}$ time point and decreased step by step over the following $24 \mathrm{~h}$ of recovery phase (Fig. 1, Suppl. Fig. S7).

\subsection{Metabolic flow in the recovery phase}

We additionally analysed carbon redistribution during the recovery phase (Fig. 1, Suppl. Figs. S2-S4). Many changes in isotope redistribution tended towards returning to the control values. G6P reached the control level in a stepwise manner with no difference being detected $24 \mathrm{~h}$ following removal of the stressor (Fig. 1, Suppl. Fig. S2). The ${ }^{13} \mathrm{C}$-redistribution to pyruvate in menadione treated plants decreased to the control level at the end of stress phase. It further decreased at $24 \mathrm{~h}$ but came back again to the control level at $30 \mathrm{~h}$. The label redistribution to citrate, 2OG, succinate and malate decreased continuously until $24 \mathrm{~h}$ in the menadione treated plants despite the removal of stressor. However it stopped to decrease or slightly increased at $30 \mathrm{~h}$. The label redistribution to fumarate was reduced through the recovery phase (Fig. 1, Suppl. Fig. S3). The ${ }^{13} \mathrm{C}$-redistribution in some amino acids such as glutamate, aspartate and tyrosine displayed changes similar to those of the TCA cycle intermediates such as citrate. By contrast, the ${ }^{13} \mathrm{C}$-redistribution in serine in stressed plants remained lower than that of control throughout the recovery phase. The ${ }^{13} \mathrm{C}$-redistribution of OAS was, however, reduced following the removal of menadione and reached the control level at $30 \mathrm{~h}$. The ${ }^{13} \mathrm{C}$ redistribution to ornithine similarly increased on the application of stress and decreased during the recovery phase (Fig. 1, Suppl. Fig. S4).

\subsection{Enzyme activity changes}

We next measured the total cellular activities of key enzymes associated to glycolysis (Fig. 2) and the TCA cycle (Fig. 3) since changes in these may account for the rapid changes in metabolite abundance and isotope redistribution. The activities of the TCA cycle enzymes were additionally determined in the recovery phase since the TCA cycle metabolite levels and isotope labelling pattern were strongly affected throughout the time course of the experiment (Fig. 3). The following enzymes of the glycolytic pathway were analysed: PGI, PFK, TPI, GAPDH, PGK, ENO and PK. In addition the enzymes of the TCA cycle: CSY, IDH, ACO and $\mathrm{MDH}$, were investigated. Among all tested enzymes only ACO, FUM and TPI activities were altered under menadione induced stress (Figs. 2, 3). The activity of TPI was increased significantly following $6 \mathrm{~h}$ of oxidative stress. The total activity of ACO was strongly reduced following $2 \mathrm{~h}$ of stress application and remained depressed even after the menadione was removed, suggesting no recovery of ACO activity in the present study (Fig. 3). It has been shown that the ACO is very sensitive to ROS and inactivated rapidly (Verniquet et al. 1991). The activity of FUM decreased after $6 \mathrm{~h}$ of oxidative stress and recovered after the removal of menadione. The inhibition of FUM by oxidative stress was previously demonstrated in Arabidopsis seedling cultures (Obata et al. 2011).

\section{Discussion}

\section{$4.1{ }^{13} \mathrm{C}$-redistribution analysis provided new insight} into the metabolic regulation under oxidative stress

${ }^{13} \mathrm{C}$-redistribution analysis was performed to extend our understanding of menadione driven changes and their recovery. In contrast to the fractional flux analysis performed in the previous study (Lehmann et al. 2009), this carbon flow analysis at the single metabolite level appears to have uncovered novel metabolic regulation as well as re-enforcing the results from our previous metabolic profiling studies. One of the novel findings that arose here is that the decrease of carbon flux through whole TCA cycle intermediates in contrast to the metabolite abundance showed a different response at the decarboxylating and 


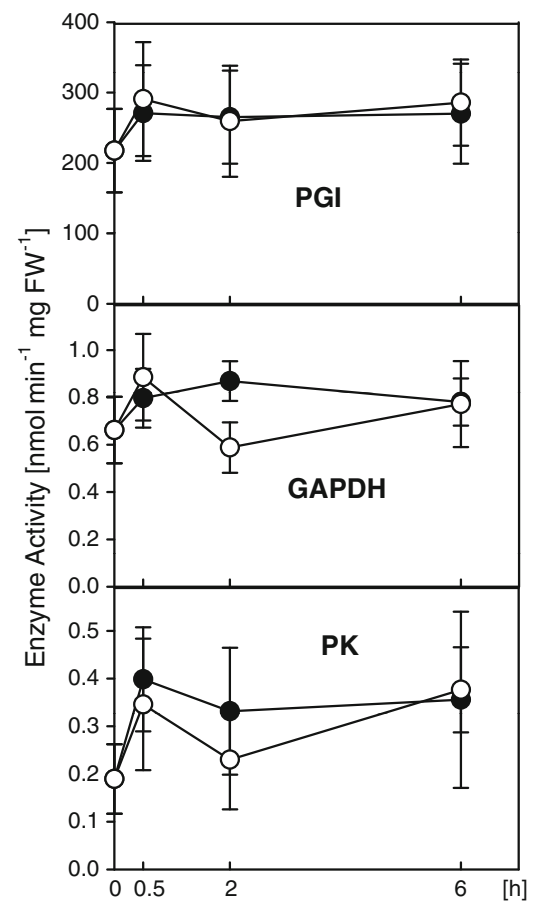

Fig. 2 Enzyme activity of glycolytic enzymes during menadione induced oxidative stress. Arabidopsis roots were treated with menadione and enzyme activities of phosphoglucose isomerase (PGI), phosphofructo kinase (PFK), triosophosphate isomerase (TPI), glyceraldehyde-3-phosphate dehydrogenase (GAPDH), 3-phospho-glycerate
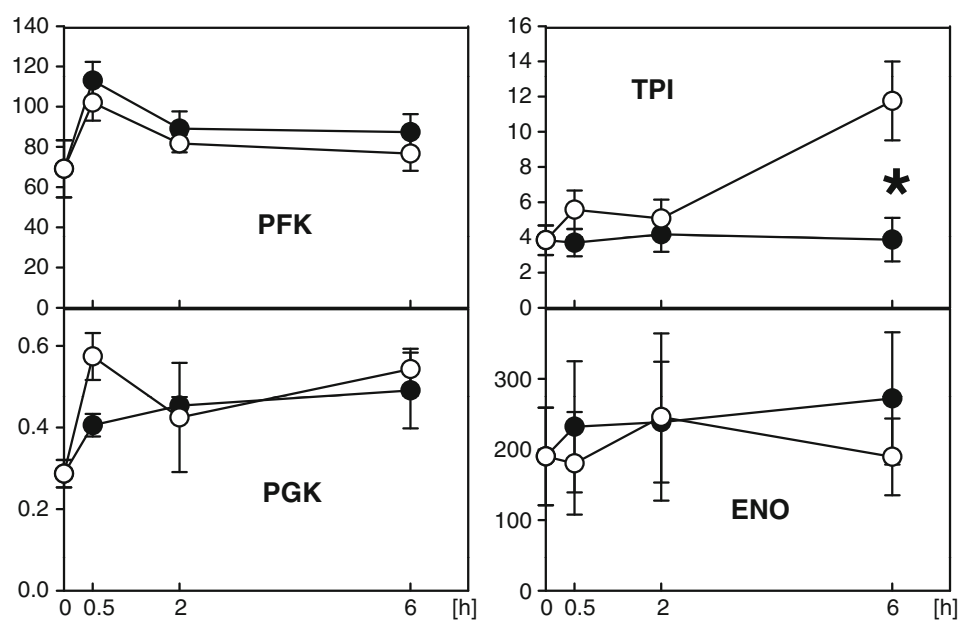

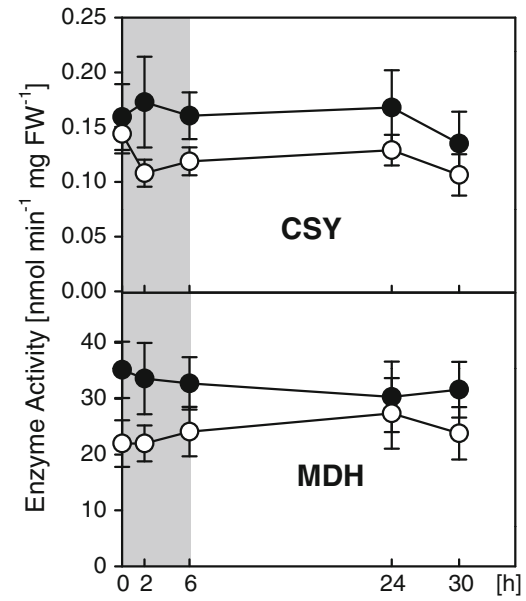

Fig. 3 Enzyme activity measurements of TCA cycle enzymes during menadione induced oxidative stress and recovery. Arabidopsis roots were treated with menadione for $6 \mathrm{~h}$ (grey box) and then the menadione was removed from growth media. The time points 24 and $30 \mathrm{~h}$ characterising the response after the menadione removal. The enzyme activities of citrate synthase (CSY), $\mathrm{NAD}^{+}$dependent kinase (PGK), enolase and pyruvate kinase (PK) in whole cell extract were measured. Menadione treated samples are open symbols and the control samples are the closed symbols. The values are means of four biological replicates \pm SEM. Significant changes were evaluated by using $t$-test $(P<0.05)$ and characterised by asterisk non-decarboxylating parts of the cycle (Lehmann et al. 2009). This indicates the down-regulation of the cyclic flux-mode of TCA cycle (Sweetlove et al. 2010) under the oxidative stress. It is well known that TCA cycle enzymes

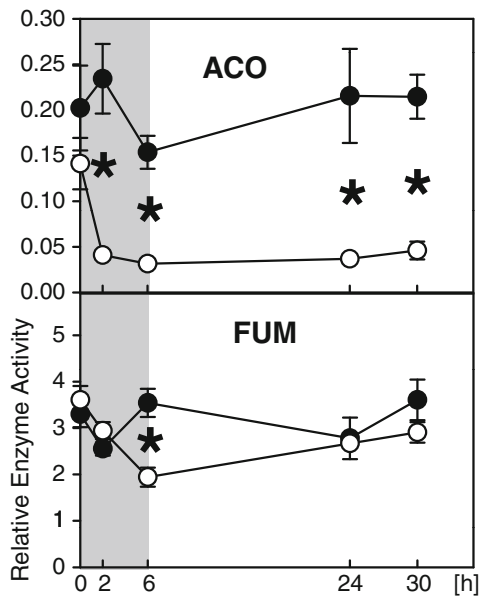

isocitrate dehydrogenase (IDH), aconitase (ACO), malate dehydrogenase $(\mathrm{MDH})$ and fumarase (FUM) in whole cell extract were measured. Menadione treated samples are open symbols and the control samples are the closed symbols. The values are the means of six biological replicates \pm SEM. Significant changes were evaluated by using $t$-test $(P<0.05)$ and shown by asterisk

such as $\mathrm{ACO}$, pyruvate-dehydrogenase (PDH) and 2-oxoglutarate-dehydrogenase are sensitive to oxidative stress (Baxter et al. 2007a; Sweetlove et al. 2002; Verniquet et al. 1991). In this study we demonstrated a strongly reduced 
activity of ACO and FUM as also reported in Arabidopsis seedlings under menadione treatment (Obata et al. 2011) This deficit in enzymatic activity could well explain the lower carbon flow through the cycle since it is shown in cardiomycytes that even only the loss of ACO activity by oxidative stress is sufficient to jeopardize the whole TCA cycle activity (Janero and Hreniuk 1996). The increased amount of citrate and $2 \mathrm{OG}$ are probably the consequence of alteration of other metabolic pathways such as $\beta$-oxidation and amino acid metabolism, respectively. On the other hand, the results of carbon flow analysis were consistent with the metabolite abundance in most pathways. For instance, the down-regulation of the glycolytic pathway within the first stress period was confirmed by the carbon flow analysis. The reduced label redistribution of the hexose phosphates suggested a reduced carbon flow through the pathway under oxidative stress. Furthermore, the accumulation of the ${ }^{13} \mathrm{C}$ label in the pyruvate pool suggested an inhibition of downstream reaction such as the TCA cycle, also suggested in this study.

It should be mentioned that the ${ }^{13} \mathrm{C}$-redistribution to pyruvate and OAS must be interpreted with caution. Both metabolites accumulated to extremely high levels in the stressed samples (Lehmann et al. 2009), and this may render them particularly susceptible to artefacts caused by dilution effects (see Geigenberger et al. 1997 for details). That said, it is clear that the carbon flow into these metabolites increased and recovered during stress and recovery phase, respectively.

\subsection{The root metabolism recovered from the oxidative} stress in the pathway-specific manner

Most metabolites showed a recovery response after the removal of menadione. This suggests that the oxidative stress did not kill the plant after $6 \mathrm{~h}$ and that a large proportion of this response must be actively regulated and furthermore not be the effect of irreversible changes driven by oxidative damage. It is demonstrated that menadione causes a long-lasting redox imbalance in Arabidopsis roots, which induce a higher percentage of cell death (Lehmann et al. 2009). Our results here, however, demonstrated that plant metabolism can adequately and effectively recover from such a relatively severe oxidative stress.

Interestingly, the time needed to recover from the oxidative stress was distinct in each pathway. This could reflect the nature of the regulatory mechanism underlying the metabolic regulation. For instance, very fast changes of glycolytic intermediates after menadione application are observed in the previous study (Lehmann et al. 2009) and it was re-arranged very quickly after the removal of menadione. These results suggest active down-regulation and re-activation of glycolysis. Furthermore the lack of inhibition of glycolytic enzymes suggested that the regulatory mechanism does not depend on the regulation of the levels of enzymes. Glycolytic enzymes have been proven to be functionally associated with the mitochondrial outer surface (Giegé et al. 2003). The association responds to respiratory demand and supports the complex formation of glycolytic enzymes leading to metabolite channeling (Graham et al. 2007). Interestingly TPI and GAPDH are more associated to mitochondria under menadione-induced oxidative stress (Obata et al. 2011). These data strongly suggest that glycolytic activity is regulated under oxidative stress by the rearrangement of glycolytic enzyme complexes on the mitochondrial outer surface allowing quick responses to the environmental changes. In the recovery process transcriptional regulation is likely also to be involved since both the accumulation of the glycolytic transcripts (Lehmann et al. 2009) and activation of TPI activity were observed following $6 \mathrm{~h}$ of stress. Transcriptional activation of glycolytic enzymes by ROS stimulation is reported also in hepatoma cells (Shi et al. 2009) and it is likely to enhance the re-activation of glycolysis.

On the other hand, the TCA cycle recovered after the menadione removal much slower than the glycolysis. A rapid stress and recovery response were detected in the levels of malate but $2 \mathrm{OG}$, succinate and fumarate took more than $24 \mathrm{~h}$ to reach their basal level despite showing a clear tendency of recovery throughout the experiment. These longer periods for recovery suggested that the regulation of TCA cycle is largely dependent on the irreversible inactivation on enzyme activities which take a long time to repair or even require de novo synthesis. Actually the ACO activity was inhibited strongly and quickly after the oxidative stress induction but did not show any recovery. The oxidative inhibition of ACO is related to the inactivation and release of $\mathrm{Fe}-\mathrm{S}$ reaction centre by ROS (Flint et al. 1993). The biogenesis of iron-sulphur cluster requires complex metabolic systems and relocation of cysteine and iron (Xu and Møller 2008) and would take a long time. It is also considerable that the slow recovery of the TCA cycle is due to the oxidation of mitochondrial matrix. Schwarzlander et al. (2009) investigated the redox state of each cellular compartment of Arabidopsis roots following menadione treatment and demonstrated that the mitochondria recovered from the oxidation much slower than the cytosol and the plastid. The TCA cycle enzymes localised in the mitochondria should be suffered from the oxidative environment longer than glycolytic enzymes in the cytosol. Nevertheless, the inhibition of the FUM activity recovered after the stress was removed. This finding correlated to the recovery response of malate and suggested a weaker effect of ROS on this part of the TCA cycle. However, the steady inhibition of the decarboxylating part of the TCA cycle might prohibit a proper activation of whole cycle. 
Despite the above hypothesis being our favoured one we cannot formally eliminate that the behaviour of isotope may partially reflect the complex kinetic relationships within the metabolic network. Modeling of metabolic flux would potentially provide such evidence, however, it is currently impossible to model a non-steady-state system such that analysed here (Baxter et al. 2007b). However, it is most likely that the ${ }^{13} \mathrm{C}$-redestribution reflects more the status of mainstream pathway such as glycolysis and the TCA cycle than the complex kinetic properties of the network since the independent metabolites in these pathways behave in a similar manner especially in the recovery phase.

\subsection{Metabolic regulation of respective pathways} following the secession of oxidative stress

A redirection of carbon flow from glycolysis into the OPPP has been previously reported following oxidative stress in Arabidopsis cell cultures (Baxter et al. 2007a) and yeast (Shenton and Grant 2003). It is considered to provide NADPH consumed by ROS scavenging and might be mediated by the inactivation of specific enzymes such as ENO, GAPDH and alcohol dehydrogenase in yeast (Shenton and Grant 2003). The OPPP intermediates accumulated in Arabidopsis roots under oxidative stress in the previous (Lehmann et al. 2009) and the present study indicate a similar metabolic regulation. These metabolites showed little recovery response but rather maintained a higher level following the removal of menadione whereas the OPPP transcripts were decreased after $6 \mathrm{~h}$ of menadione treatment in the previous study (Lehmann et al. 2009). These results suggest that the reduction of OPPP activity would be achieved mainly by transcriptional regulation which acts across a longer timeframe than the post-translational activation of glycolysis.

Citrate accumulated continuously throughout the time course even in the recovery phase. The accumulation of citrate under stress conditions is well known in plants. For example Luettge (1990) reported an accumulation of citrate in the Goethe plant, Kalanchoe pinnata (Lam.), in response to irradiation, mineral nutrition and drought stress. The huge accumulation of citrate, observed in this study, did not accompany any changes in CSY activity implying that the citrate may be synthesised through other metabolic pathways than the TCA cycle, a hypothesis supported by results of our ${ }^{13} \mathrm{C}$ redistribution studies.

A general increase of amino acid levels following oxidative stress has been reported previously (Lehmann et al. 2009) and is thought to be the result of enhanced protein degradation since oxidative stress and protein oxidation are known to promoted this process (Friguet 2006; Møller et al. 2007; Palma et al. 2002). However, the isotope redistribution was only observed to be altered in specific amino acids in the present study. That said we cannot rule out that some of these (lack of) changes could be due to the metabolic effect of glucose supply (see for example Mishra et al. 2009; Hesse et al. 2003) particularly given that it has been reported that sugars may protect plants from oxidative stress (Ramel et al. 2009).

OAS is a key intermediate of the sulphur assimilation pathway, which is known to respond to stress (Kawashima et al. 2005; Saito 2004). OAS is accumulated under sulphur limitation and act as a signal indicating sulphur/ nitrogen availability in plants (Hawkesford and De Kok 2006; Kim et al. 1999). In the present study OAS displayed rapid responses to stress and also its cessation. It seems likely that cellular oxidation, induced by menadione, inhibited sulphur assimilation and caused OAS accumulation (Scheerer et al. 2010). The recovery of OAS might reflect a status of normalised nutrition following the re-reduction of the cellular glutathione pool following the removal of menadione (Schwarzlander et al. 2009). The re-activation of nitrogen assimilation is suggested by the recovery of glutamate and aspartate metabolism since these amino acids are directly related to the nitrogen assimilation. Additionally the high accumulation of OAS, itself, could activate sulphur assimilation and accelerate the recovery from the oxidative stress (Hawkesford and De Kok 2006).

\section{Concluding remarks}

Plant root metabolism was rapidly altered under oxidative stress but recovered when the stress was removed. A quick response of particular metabolites such as hexose phosphates and pyruvate in both stress and recovery phase would enable them to act as a key metabolites for the reconfiguration of metabolic network. Such key metabolites could be tangible targets for metabolic engineering of stress tolerant plants. Furthermore, the results suggest the involvement of distinctive regulatory mechanisms for individual pathways of central metabolism such as the glycolysis and the TCA cycle. The remarkable ability of the glycolysis to be reactivated appears to be achieved by a regulatory mechanism uncoupled from the regulation of enzymatic activity. Such a response is very beneficial for plant root metabolism since it ensures both energy production and supply of reducing equivalents and demonstrates the importance of the metabolic recovery following the secession of stresses for plant survival.

Acknowledgments The work was funded by the ERA-Plant Genomics grant to A.R.F. Thanks to Jan Hüge and Joachim Kopka for help in the ${ }^{13} \mathrm{C}$ label calculation. 
Open Access This article is distributed under the terms of the Creative Commons Attribution Noncommercial License which permits any noncommercial use, distribution, and reproduction in any medium, provided the original author(s) and source are credited.

\section{References}

Apel, K., \& Hirt, H. (2004). Reactive oxygen species: Metabolism, oxidative stress, and signal transduction. Annual Review of Plant Biology, 55, 373-399.

Bai, J., Gong, C. M., Chen, K., Kang, H. M., \& Wang, G. (2009). Examination of antioxidative system's responses in the different phases of drought stress and during recovery in desert plant Reaumuria soongorica (Pall.) Maxim. Journal of Plant Biology, 52, 417-425.

Baxter, C. J., Liu, J. L., Fernie, A. R., \& Sweetlove, L. J. (2007a). Determination of metabolic fluxes in a non-steady-state system. Phytochemistry, 68, 2313-2319.

Baxter, C. J., Redestig, H., Schauer, N., et al. (2007b). The metabolic response of heterotrophic Arabidopsis cells to oxidative stress. Plant Physiology, 143, 312-325.

Brandina, I., Graham, J., Lemaitre-Guillier, C., et al. (2006). Enolase takes part in a macromolecular complex associated to mitochondria in yeast. Biochemica et Biophysica Acta-Bioenergetics, 1757, 1217-1228.

Castro, F. A. V., Herdeiro, R. S., Panek, A. D., Eleutherio, E. C. A., \& Pereira, M. D. (2007). Menadione stress in Saccharomyces cerevisiae strains deficient in the glutathione transferases. Biochimica et Biophysica Acta-General Subjects, 1770, 213-220.

Chung, S. H., Chung, S. M., Lee, J. Y., et al. (1999). The biological significance of non-enzymatic reaction of menadione with plasma thiols: Enhancement of menadione-induced cytotoxicity to platelets by the presence of blood plasma. FEBS Letters, 449, 235-240.

Fait, A., Fromm, H., Walter, D., Galili, G., \& Fernie, A. R. (2008). Highway or byway: The metabolic role of the GABA shunt in plants. Trends in Plant Science, 13, 14-19.

Fell, D. A. (2005). Enzymes, metabolites and fluxes. Journal of Experimental Botany, 56, 267-272.

Fernie, A. R., Geigenberger, P., \& Stitt, M. (2005). Flux an important, but neglected, component of functional genomics. Current Opinion in Plant Biology, 8, 174-182.

Flint, D. H., Tuminello, J. F., \& Emptage, M. H. (1993). The inactivation of Fe-S cluster containing hydro-lyases by superoxide. The Journal of Biological Chemistry, 268, 22369-22376.

Friguet, B. (2006). Oxidized protein degradation and repair in ageing and oxidative stress. FEBS Letters, 580, 2910-2916.

Geigenberger, P., Reimholz, R., Geiger, M., et al. (1997). Regulation of sucrose and starch metabolism in potato tubers in response to short-term water deficit. Planta, 201, 502-518.

Gibeaut, D. M., Hulett, J., Cramer, G. R., \& Seemann, J. R. (1997). Maximal biomass of Arabidopsis thaliana using a simple, lowmaintenance hydroponic method and favorable environmental conditions. Plant Physiology, 115, 317-319.

Gibon, Y., Blaesing, O. E., Hannemann, J., et al. (2004). A robotbased platform to measure multiple enzyme activities in Arabidopsis using a set of cycling assays: Comparison of changes of enzyme activities and transcript levels during diurnal cycles and in prolonged darkness. Plant Cell, 16, 3304-3325.

Giegé, P., Heazlewood, J. L., Roessner-Tunali, U., et al. (2003). Enzymes of glycolysis are functionally associated with the mitochondrion in Arabidopsis cells. Plant Cell, 15, 2140-2151.
Gong, Q., Li, P., Ma, S., Indu Rupassara, S., \& Bohnert, H. J. (2005). Salinity stress adaptation competence in the extremophile Thellungiella halophila in comparison with its relative Arabidopsis thaliana. Plant Journal, 44, 826-839.

Graham, J. W. A., Williams, T. C. R., Morgan, M., et al. (2007). Glycolytic enzymes associate dynamically with mitochondria in response to respiratory demand and support substrate channeling. Plant Cell, 19, 3723-3738.

Hawkesford, M. J., \& De Kok, L. J. (2006). Managing sulphur metabolism in plants. Plant Cell and Environment, 29, 382-395.

Hesse, H., Trachsel, N., Suter, M., et al. (2003). Effect of glucose on assimilatory sulphate reduction in Arabidopsis thaliana roots. Journal of Experimental Botany, 54, 1701-1709.

Janero, D. R., \& Hreniuk, D. (1996). Suppression of TCA cycle activity in the cardiac muscle cell by hydroperoxide-induced oxidant stress. American Journal of Physiology-Cell Physiology, 270, C1735-C1742.

Jenner, H. L., Winning, B. M., Millar, A. H., et al. (2001). NAD malic enzyme and the control of carbohydrate metabolism in potato tubers. Plant Physiology, 126, 1139-1149.

Kaplan, F., Kopka, J., Sung, D. Y., et al. (2007). Transcript and metabolite profiling during cold acclimation of Arabidopsis reveals an intricate relationship of cold-regulated gene expression with modifications in metabolite content. Plant Journal, 50, 967-981.

Kawashima, C. G., Berkowitz, O., Hell, R., Noji, M., \& Saito, K. (2005). Characterization and expression analysis of a serine acetyltransferase gene family involved in a key step of the sulfur assimilation pathway in Arabidopsis. Plant Physiology, 137, 220-230.

Kim, H., Hirai, M. Y., Hayashi, H., Chino, M., Naito, S., \& Fujiwara, T. (1999). Role of $O$-acetyl-L-serine in the coordinated regulation of the expression of a soybean seed storage-protein gene by sulfur and nitrogen nutrition. Planta, 209, 282-289.

Lehmann, M., Schwarzländer, M., Obata, T., et al. (2009). The metabolic response of Arabidopsis roots to oxidative stress is distinct from that of heterotrophic cells in culture and highlights a complex relationship between the levels of transcripts, metabolites, and flux. Molecular Plant, 2, 390-406.

Lisec, J., Schauer, N., Kopka, J., Willmitzer, L., \& Fernie, A. R. (2006). Gas chromatography mass spectrometry-based metabolite profiling in plants. Nature Protocols, 1, 387-396.

Luettge, U. (1990). Nocturnal citrate accumulation and its response to environmental stress in the CAM Plant Kalanchoe-Pinnata (Lam.) Pers. Plant Cell and Environment, 13, 977-982.

Mauzeroll, J., Bard, A. J., Owhadian, O., \& Monks, T. J. (2004). Menadione metabolism to thiodione in hepatoblastorna by scanning electrochemical microscopy. Proceedings of the National Academy of Sciences of the United States of America, 101, 17582-17587.

Mishra, B. S., Singh, M., Aggrawal, P., \& Laxmi, A. (2009). Glucose and auxin signaling interaction in controlling Arabidopsis thaliana seedlings root growth and development. PLoS One, 4, e4502.

Møller, I. M., Jensen, P. E., \& Hansson, A. (2007). Oxidative modifications to cellular components in plants. Annual Review of Plant Biology, 58, 459-481.

Obata, T., Matthes, A., Koszior, S., et al. (2011). Alteration of mitochondrial protein complexes in relation to metabolic regulation under short-term oxidative stress in Arabidopsis seedlings. Phytochemistry (published online). doi:10.1016/ j.phytochem.2010.11.003.

Palma, J. M., Sandalio, L. M., Corpas, F. J., et al. (2002). Plant proteases, protein degradation, and oxidative stress: Role of peroxisomes. Plant Physiology and Biochemistry, 40, 521530.

Ramel, F., Sulmon, C., Bogard, M., Couee, I., \& Gouesbet, G. (2009). Differential patterns of reactive oxygen species and antioxidative 
mechanisms during atrazine injury and sucrose-induced tolerance in Arabidopsis thaliana plantlets. BMC Plant Biology, 9, 28.

Roessner, U., Luedemann, A., Brust, D., et al. (2001). Metabolic profiling allows comprehensive phenotyping of genetically or environmentally modified plant systems. Plant Cell, 13, 11-29.

Roessner-Tunali, U., Liu, J. L., Leisse, A., et al. (2004). Kinetics of labelling of organic and amino acids in potato tubers by gas chromatography-mass spectrometry following incubation in C-13 labelled isotopes. Plant Journal, 39, 668-679.

Saito, K. (2004). Sulfur assimilatory metabolism. The long and smelling road. Plant Physiology, 136, 2443-2450.

Scheerer, U., Haensch, R., Mendel, R. R., et al. (2010). Sulphur flux through the sulphate assimilation pathway is differently controlled by adenosine $5^{\prime}$-phosphosulphate reductase under stress and in transgenic poplar plants overexpressing gamma-ECS, SO, or APR. Journal of Experimental Botany, 61, 609-622.

Schutzendubel, A., \& Polle, A. (2002). Plant responses to abiotic stresses: Heavy metal-induced oxidative stress and protection by mycorrhization. Journal of Experimental Botany, 53, 1351-1365.

Schwarzlander, M., Fricker, M. D., \& Sweetlove, L. J. (2009). Monitoring the in vivo redox state of plant mitochondria: Effect of respiratory inhibitors, abiotic stress and assessment of recovery from oxidative challenge. Biochemica et Biophysica Acta-Bioenergetics, 1787, 468-475.

Shenton, D., \& Grant, C. M. (2003). Protein S-thiolation targets glycolysis and protein synthesis in response to oxidative stress in the yeast Saccharomyces cerevisiae. Biochemical Journal, 374, 513-519.
Shi, D. Y., Xie, F. Z., Zhai, C., et al. (2009). The role of cellular oxidative stress in regulating glycolysis energy metabolism in hepatoma cells. Molecular Cancer, 8, 32.

Shulaev, V., Cortes, D., Miller, G., \& Mittler, R. (2008). Metabolomics for plant stress response. Physiologia Plantarum, 132, 199-208.

Sweetlove, L. J., Beard, K. F. M., Nunes-Nesi, A., Fernie, A. R., \& Ratcliffe, R. G. (2010). Not just a circle: Flux modes in the plant TCA cycle. Trends in Plant Science, 15, 462-470.

Sweetlove, L. J., Heazlewood, J. L., Herald, V., et al. (2002). The impact of oxidative stress on Arabidopsis mitochondria. Plant Journal, 32, 891-904.

Usadel, B., Nagel, A., Thimm, O., et al. (2005). Extension of the visualization tool MapMan to allow statistical analysis of arrays, display of corresponding genes, and comparison with known responses. Plant Physiology, 138, 1195-1204.

Van den Ende, W., \& Valluru, R. (2009). Sucrose, sucrosyl oligosaccharides, and oxidative stress: Scavenging and salvaging? Journal of Experimental Botany, 60, 9-18.

Verniquet, F., Gaillard, J., Neuburger, M., \& Douce, R. (1991). Rapid inactivation of plant aconitase by hydrogen peroxide. Biochemical Journal, 276, 643-648.

Virtanen, A. I. (1961). Prefatory chapter some aspects of amino acid synthesis in plants and related subjects. Annual Review of Plant Physiology, 12, 1-13.

$\mathrm{Xu}, \mathrm{X}$. M., \& Møller, S. G. (2008). Iron-sulfur cluster biogenesis systems and their crosstalk. ChemBioChem, 9, 2355-2362. 